\title{
Malabsorption of Oral Antibiotics in Pregnancy after Gastric Bypass Surgery
}

\author{
Susanna R. Magee, MD, MPH, Grace Shih, MD, and Anne Hume, PharmD
}

Gastric bypass surgery, by definition, changes the absorption capabilities of the stomach and small intestine. The use of oral medications in patients post gastric bypass may need to be adjusted by medical providers to account for this absorption change. The following case exemplifies this dilemma in a pregnant patient status post gastric bypass surgery with a complicated urinary tract infection. ( $\mathrm{J}$ Am Board Fam Med 2007;20:310-313.)

The patient is a 29-year-old G2 P0101 who presented for prenatal care at 9 weeks gestation dated by last menstrual period and first trimester ultrasound. Her past medical history is complicated by morbid obesity, for which she underwent a Roux-en-Y gastric bypass in 2003. She tolerated the procedure well, and aside from a 2-week hospital stay after the surgery in 2003, she has been free of complications.

Her obstetrical history is notable for an emergent cesarean section in 1996 after developing eclampsia at 32 weeks gestation. The patient did well post cesarean section, and her now 10-year-old child has no complications from prematurity.

Before her pregnancy, she was taking only vita$\min B_{12}$ and iron supplementation in oral form. At her first visit with the practice nurse (scheduled to confirm the diagnosis of pregnancy), it was noted that her urinalysis was positive for nitrites and leukocyte esterase. She also had $2+$ protein in her bedside urinalysis. The patient was asymptomatic; however, it was presumed from this urinalysis that the patient had a urinary tract infection. The covering physician was consulted, and the patient was

This article was externally peer reviewed.

Submitted 12 October 2006; revised 1 December 2006; accepted 12 February 2007.

From Brown Medical School, Providence, RI (SRM, GS), Memorial Hospital of Rhode Island, Pawtucket, RI (SRM, GS); and University of Rhode Island, Kingston, RI (AH).

Funding: none.

Conflict of interest: none declared.

Corresponding author: Susanna R. Magee, MD, MPH, Director of Maternal and Child Health, Brown University Department of Family Medicine, Family Care Center, Memorial Hospital at Rhode Island, 111 Brewster Street, Pawtucket, RI 02860 (E-mail: Susanna_Magee@mhri.org). treated with oral amoxicillin. Two days later, the culture grew out $>100,000$ colonies of Escherichia coli. Sensitivity testing showed that the organism was sensitive to amoxicillin that had been prescribed.

Two weeks later, the patient presented for her initial obstetrical examination. Once again, despite reported patient compliance, the urinalysis was nitrite, protein, and leukocyte esterase positive. The patient was treated this time with macrodantin. Two days later, the culture showed $>100,000$ colonies of $E$. coli, and the specimen was found to be sensitive to both amoxicillin and marcodantin.

The physician called the patient to ensure, once again, that she was compliant. She had not missed any doses. The patient was employed as a registered nurse in the outpatient setting and was fully aware of the necessity of compliance with all medications, especially antibiotic regimens.

Two days later, the patient developed nausea, vomiting, fever to $102^{\circ} \mathrm{F}$, and CVA tenderness on the right. She was sent to the hospital to be admitted for presumed pyelonephritis. Ultrasound of the kidney showed a small $(<1 \mathrm{~cm})$ nonobstructing right renal stone in the inferior pole. After 24 hours of ceftriaxone, the patient was afebrile and felt well. Urology was consulted on hospital day 2, and despite the failure of 2 outpatient regimens of oral antibiotic that were appropriate choices in terms of sensitivities, the specialist recommended amoxicillin/clavulanate as prophylaxis at $500 \mathrm{mg}$ daily.

The patient was sent home on this regimen. Five days later, a test of cure analysis showed a normal urinalysis and a culture showed no growth. 
After 10 days on this regimen, the patient developed fever and costovertebral angle tenderness again and was hospitalized for a second case of pyelonephritis with a pan-sensitive E. coli. She was treated with intravenous ceftriaxone for 48 hours. After much discussion regarding prophylaxis medications with maternal fetal medicine specialists, obstetricians and family physicians, the decision was made to use weekly intramuscular ceftriaxone as prophylaxis.

The patient remained infection free from that point on and went on to deliver by elective repeat cesarean section at 39 weeks gestation.

\section{Background}

Obesity, defined as a body mass index (BMI) of greater than $30 \mathrm{~kg} / \mathrm{m}^{2}$, is a growing epidemic in the United States. ${ }^{1}$ The prevalence of obesity is more than $18 \%$ and continues to increase across all sociodemographic groups. This epidemic has a profound impact on our nation's health, as obesity is associated with many medical problems, including diabetes mellitus, hypertension, coronary heart disease, and cerebral stroke. Researchers have estimated that the annual number of deaths attributable to obesity is roughly $280,000 .^{2}$

Obesity has particular importance to women's health because of its significant impact on the reproductive system. Obesity has been linked to menstrual abnormality, infertility, and miscarriage. In pregnant women, obesity is associated with an increased risk of gestational diabetes mellitus, gestational hypertension, preeclampsia, eclampsia, fetal macrosomia, and anesthesia complications. ${ }^{3}$

Treatment of obesity is complicated. Although diet and exercise may work for some patients, studies have shown that bariatric surgery is an effective method of weight loss, especially in morbidly obese patients. ${ }^{4-6}$ Although there are several types of bariatric surgery, this case report focuses on the most common type of restrictive-malabsorptive surgery, the Roux-en-Y gastric bypass. In this surgery, the fundus of the stomach is surgically separated or removed. Then, a gastrojejunostomy is performed allowing the stomach contents to empty into the distal jejunum. Contents of the excluded stomach, duodenum, and proximal jejunum empty into the intestinal tract distally. Patients lose weight by consuming fewer calories because of the decreased capacity of the stomach pouch. ${ }^{7}$
After gastric bypass surgery, women may experience several complications. If they fail to reduce food intake, they may experience nausea and vomiting. Outlet obstruction may occur from poorly chewed food or strictures at the stoma. Early dumping syndrome occurs from accelerated gastric emptying of hyperosmolar contents into the small bowel. Because of the contents' hyperosmolarity, fluid moves rapidly from the intravascular space into bowel lumen, causing distention and increased peristalsis. Patients may experience crampy abdominal pain, bloating, or diarrhea. The contraction of intravascular space may cause tachycardia and lightheadedness. Late dumping syndrome occurs 1 to 3 hours after a meal and is due to the hyperinsulenimic response. This leads to subsequent hypoglycemia. ${ }^{8}$

Nutrient deficiencies are common after gastric bypass surgery. ${ }^{9,10}$ The most commonly seen nutrient deficiencies are iron, vitamin $\mathrm{B}_{12}$, folate, and calcium deficiencies. Iron deficiency occurs because of decreased interaction of food contents with gastric acid, which converts iron from the insoluble ferrous form to the more soluble ferric form. Vitamin $B_{12}$ levels are decreased because of lack of contact with intrinsic factor, which is necessary for absorption. Folate and calcium/vitamin D deficiencies occur because the duodenum is bypassed, the primary site of absorption for these nutrients.

Morbid obesity presents many challenges in the provision of optimal drug therapy. Traditionally clinical trials have excluded people with significant obesity leaving clinicians to dose medications such as low molecular weight heparins and antibiotic agents based on small, limited pharmacokinetic studies. For people who are morbidly obese undergoing gastric bypass, recent data have also indicated that standard prophylactic antibiotic regimens with cefazolin may not result in adequate therapeutic tissue levels especially in people with a BMI greater than $50 \mathrm{~kg} / \mathrm{m}^{2} .{ }^{11}$ A single-dose study using erythromycin base demonstrated a reduction of approximately $50 \%$ in the peak serum concentration after bariatric surgery. ${ }^{12}$

After gastric bypass surgery, new considerations develop with respect to drug therapy. Decreased intestinal length leads to reduced time available for drug absorption which is important in extended release preparations. ${ }^{11}$ In addition, the reduction in the size of the gastric pouch has caused many bariatric surgeons to recommend that nonsteroidal an- 
ti-inflammatory drugs be avoided permanently post gastric bypass, because of a potentially increased risk of ulceration. ${ }^{12,13}$ Bypassing parts of the small intestine decreases drug contact with villi, which decreases the surface area available for absorption. Finally, drug solubility may be altered because of the changes in gastric $\mathrm{pH}$ post bypass surgery. ${ }^{14}$ Some studies suggest that there is variability in drug dissolution due to the gastric environment post bypass surgery. ${ }^{15}$ In general, oral solutions and rapid-release drug formulations would be preferred over sustained release products.

Little information is available regarding the absorption of oral antibiotics commonly used in the treatment and prophylaxis of urinary tract infections due to $E$. coli in people who have had prior gastric bypass surgery. Among these antibiotics are penicillins, cephalosporins, quinolones, sulfamethoxazole, nitrofurantoin, and macrolides. Penicillin antibiotics vary greatly in their susceptibility to the effect of gastric acid and food on their absorption with amoxicillin generally having more than $70 \%$ absorption and peak drug levels 2 to 2.5 times higher than those with a similar dose of ampicillin. Older oral penicillins such as penicillin G are susceptible to gastric acid, and achlorhydria would be expected to increase absorption. ${ }^{18}$

Some beta-lactam antibiotics may undergo active absorption by serving as substrates for peptide transporter PEPT1 in the brush border of the small intestine. ${ }^{19}$ The extent of absorption through this pathway is variable and may be dependent on the structure of the individual antibiotic. Absorption through PEPT1 may also be $\mathrm{pH}$ and calcium dependent. Cephalosporins are acid stable and, depending on their structure, some drugs in the class including cephalexin, cefradoxil, cefaclor, and loracarbef are absorbed extensively by the intestinal PEPT1 transport system. ${ }^{18}$

Nitrofurantoin is rapidly absorbed in the upper small bowel with approximately $40 \%$ to $50 \%$ absorbed. Food has been shown to increase the absorption of nitrofurantoin. One preparation (Macrodantin) contains nitrofurantoin in a macrocrystalline formulation, which has a slower rate of dissolution and absorption, compared with the monohydrate form of the drug. This preparation might be expected to have reduced effectiveness in a person who had undergone gastric bypass. ${ }^{18}$

As a class, quinolone antibiotics are greater than $50 \%$ absorbed with levofloxacin, gatifloxacin, and moxifloxacin having almost 100\% bioavailability. The presence of food or achlorhydria does not significantly affect absorption of quinolones, although food may delay the time to peak drug concentration. Sulfamethoxazole is generally rapidly and completely absorbed from small intestine and stomach whether used alone or in combination with trimethoprim. Macrolide and ketolide antibiotics vary greatly in their absorption with erythromycin base susceptible to destruction by gastric acid. Clarithromycin is approximately $50 \%$ bioavailable, whereas absorption of azithromycin is only $37 \%$ with food decreasing its absorption by $50 \% .^{18}$

\section{Discussion}

Few specific recommendations exist regarding optimal drug therapy regimens for the person who has undergone gastric bypass. In our case, the nutritive deficiencies common to post-bypass patients were addressed by oral iron and vitamin $\mathrm{B}_{12}$ replacement. Her vitamin $B_{12}$ levels, which were in the normal range, were checked each trimester to ensure sufficient absorption. Her oral antibiotic treatments included amoxicillin, nitrofurantoin (Macrodantin), and amoxicillin/clavulate (Augmentin). It is unclear whether the bioavailability of these medications is decreased by post bypass physiology; however, the potential for this impact has been implied by previous studies. Although our patient did not have drug levels tested to ensure therapeutic levels, this is a consideration for future practice while guidelines are established. Ultimately, it was the intravenous ceftriaxone and prophylactic intramuscular injections that resolved our patient's complicated urinary tract infection, effectively eliminating the impact of her post bypass physiology.

As bariatric surgery becomes a more common treatment for morbid obesity, health professionals should expect to see an increasing number of patients post gastric bypass surgery. The impact of this surgery is great for family medicine physicians as the nutritive status and pharmacotherapy of this population will be have special considerations. Post-bypass patients require replacement of iron, vitamin $\mathrm{B}_{12}$, calcium, and folate. Their altered anatomy may impact the form of pharmacotherapy (oral capsule, tablet, suspension; intravenous, etc), choice of medication (regular versus extended release), and monitoring of therapeutic levels to ensure proper absorption. 


\section{References}

1. Mokad AH, Serdula MK, Dietz WH, et al. The spread of the obesity epidemic in the United States, 1991-1998. JAMA 1999;282:1319-22.

2. Allison DB, Fontaine KR, Manson JE, et al. Annual deaths attributable to obesity in the United States. JAMA 1999;282:1530-38.

3. Baeten JM, Bukusi EA, Lambe M. Pregnancy complications and outcomes among overweight and obese nulliparous women. Am J Public Health 2001; 91:436-40.

4. Buchwald H, Avidor Y, Braunwald E, et al. Bariatric surgery: a systematic review and meta-analysis. JAMA 2004;292:1724-37.

5. Sheiner E, Menes TS, Silverberg D, et al. Pregnancy outcome of patients with gestational diabetes mellitus following bariatric surgery. Am J Obstet Gynecol 2006;194:431-5.

6. Sheiner E, Levy A, Silverberg D, et al. Pregnancy after bariatric surgery is not associated with adverse perinatal outcome. Am J Obstet Gynecol 2004;190: 1335-40.

7. Woodard CB. Pregnancy following bariatric surgery. J Perinat Neonat Nurs 2004;18:329-40.

8. Carvajal SH, Mulvihill SJ. Postgastrectomy syndromes: dumping and diarrhea. Gastroenterol Clin North Am 1994;23:261-79.

9. Martin LF, Kathleen MF, Noran TE. Pregnancy after adjustable gastric banding. Obstet Gynecol 2000;95:927-30.
10. Burt J. Worth the weight. Adv Nurse Prac 2005; 45-7.

11. Edminston CE, Krepel C, Kelly H, et al. Perioperative antibiotic prophyllaxis in the gastric bypass patient: do we achieve therapeutic levels? Surgery 2004; 136:738-47.

12. Price RA, Pincheira JC, Mason EE, Printen KJ. Influence of bariatric surgery on erythromyscin absorption. J Clin Pharm 1984;523-7.

13. Miller AD, Smith KM. Medication and nutrient administration considerations after bariatric surgery. Am J Health-Syst Pharm 2006;63:1852-7.

14. Wolinsky I, Williams L. Nutrition in Pharmacy Practice. Washington: American Pharmaceutical Association 2002;22-54.

15. Virji A, Murr MM. Caring for patients after bariatric surgery. Am Fam Physician 2006;73:1403-8.

16. Fussy SA. The skinny on gastric bypass: what pharmacists need to know. US Pharmacist 2005;3-12.

17. Seaman JS, Bowers SP, Dixon P, et al. Dissolution of common psychiatric medications in a Roux-en-Y gastric bypass model. Psychosomatics 2005;46:250-3.

18. Mandell GL, Bennett JE, Dolin R. Principles and practice of infectious diseases. 6th ed. Elsevier; 2005 (ISBN 044306643-4)

19. Bretscheneider B, Brrandsch M, Neubert R. Intestinal transport of beta lactam antibiotics: analysis of the affinity at the $\mathrm{H}^{+}$/Peptide symporter (PEPT1), the uptake of Caco-2 cellmonolyaers and the transepithelial flux. Pharm Res 1999;16:55-61. 